\title{
Treatment Challenges in Co-infected HIV and TB Children
}

\author{
Weerawat Manosuthi, *Jurai Wongsawat \\ Departments of Medicine and *Pediatrics, Bamrasnaradura Infectious Diseases Institute, Ministry of Public Health, Nonthaburi, \\ 11000, Thailand.drweerawat@hotmail.com
}

1 pproximately 2.5 million children globally were living with human immunodeficiency virus (HIV) at the end of 2009 and more than 800 children died daily of AIDS in each year. In resourceconstrained countries, tuberculosis (TB) is the most common opportunistic infection in children with HIV infection and most HIV-infected patients with TB have relatively advanced HIV disease [1]. Moreover, both diseases together represent a deadly co-morbidity [2]. With regard to antiretroviral therapy (ART), nevirapine is recommended as a preferred drug for children who are $<3$ years old, while efavirenz is preferred for those older than 3 years because of lacking data on dosing in children $<3$ years. The pediatric nevirapine dosages (age $\geq 15$ days) are recommended at 200 and $120-150 \mathrm{mg} / \mathrm{m}^{2}$ BSA/dose twice daily in children $<8$ years and $\geq 8$ years, respectively (maximum $200 \mathrm{mg} / \mathrm{dose}$ ). The pediatric EFV dosing is adjusted related to weight band [3].

Despite growing evidence of ART is gained in children living with $\mathrm{HIV}$ and $\mathrm{TB}$, there remain a number of significant obstacles for our pediatric care which we need to address. The question of early versus delayed initiation of ART has been recently answered by randomized controlled trials in adult patients. SAPIT trial showed that all HIV and TB patients who had CD4 cell counts less than 500 cells/ $\mathrm{mm}^{3}$ should receive concurrent (integrated) TB treatment and ART [4]. A more recent study, CAMELIA trial, demonstrated that death rate in patients where ART was initiated after 2 weeks of TB treatment was significantly higher compared to those in whom ART was initiated after 8 weeks of TB treatment; although these advantages in early ART initiation was not reported in HIV-infected patients with TB meningitis [5]. The World health Organization (WHO) recommended that ART should be initiated in all children with TB, regardless of CD4, within 2-8 weeks of starting antituberculous (anti-TB) therapy. Nevertheless, one of the major concerns is that none of the studies includes pediatric patients and thus a critical need exists for studies in children.

Studies evaluating the pharmacokinetics of antiTB drugs and drug-drug interactions between antiTB drugs and antiretroviral drugs are very scarce, especially in pediatric patients. In terms of TB treatment, rifampicin forms the backbone of first line anti-TB treatment and has allowed for the use of 6month therapy for TB. However, current available rifamycins differ in their potency as CYP3A4 enzyme inducers, with rifampicin being the most potent inducer. Unfortunately, rifampicin is the only rifamycin that is available in many resourceconstrained countries, which decreases plasma level of many antiretroviral drugs including protease inhibitors and non-nucleoside reverse transcriptase inhibitors (NNRTIs), although the magnitude of effect on NNRTIs is lesser. Decreased plasma levels of antiretroviral drugs can lead to HIV treatment failure. Currently, the minimum acceptable plasma concentrations at 12 hours after dosing for efavirenz and nevirapine are 1 and $3.4 \mathrm{mg} / \mathrm{L}$, respectively [6]. The previous randomized controlled study in HIVinfected adults demonstrated that plasma nevirapine level is significantly lower in patients who are concurrently receiving nevirapine with rifampicin, when compared to efavirenz and low NNRTI 
exposure is an important independent predictive factor for subsequent HIV treatment failure [7].

The study by Shah and colleagues [8] aimed to determine factors affecting serum levels of efavirenz and nevirapine and analyzed the effect of concomitant rifampicin on nevirapine levels in pediatric Indian patients. This study has a number of important conclusions. Firstly, the result showed that concurrent rifampicin administration does not alter blood levels of nevirapine after the dose of nevirapine was increased by $20-30 \%$. Secondly, nevirapine-associated adverse effects are notably not seen with this increase in dose while nevirapine 600 mg per day was associated with a high rate of hypersensitivity syndrome in co-infected HIV and TB adult patients. Further studies need to confirm these discordant findings. Lastly, plasma levels for efavirenz in standard dose are less compromised by concomitant treatment with rifampicin than are levels for nevirapine as same as shown in previous studies in HIV-infected adults; however, the sample size is very limited. A previous study conducted by Ren $\mathrm{Y}$ and colleagues revealed some concerns for virologic failure among HIV and TB co-infected children receiving rifampicin containing regimen and had low efavirenz levels [9]. This emphasizes the need for more studies. It would have been more interesting to know the long-term outcome in such children.

Another concern is that NNRTIs have demonstrated inter-individual and inter-racial pharmacokinetic variability because polymorphisms of CYP2B6 and CYP3A4 are associated with altered activity of hepatic iso-enzyme and resulting in a variation of plasma NNRTI level, treatment efficacy and toxicity [10]. Therefore, this result might not be applicable to all other ethnic populations and close monitoring for liver toxicity is still needed. In addition, strategy of lead-in dosing of nevirapine needs to be further explored.
Funding: None

Competing interests: None stated.

\section{REFERENCES}

1. Sterling TR, Pham PA, Chaisson RE. HIV infection-related tuberculosis: clinical manifestations and treatment. Clin Infect Dis. 2010;50 Suppl 3:S223-30.

2. Caritas.org. HAART for children: HIV/TB pediatric campaign. Available athttp://www.caritas.org/activities/ hiv_aids/the_campaign.html. Accessed on August 31, 2011 .

3. Panel on antiretroviral therapy and medical management of HIV-infected children. Guidelines for the use of antiretroviral agents in pediatric HIV infection. . Available at http://aidsinfo.nih.gov/ContentFiles/Pediatric Guide lines.pdf.Accessed on September 9, 2011.

4. Abdool Karim SS, Naidoo K, Grobler A, Padayatchi N, Baxter C, Gray A, et al. Timing of initiation of antiretroviral drugs during tuberculosis therapy. $\mathrm{N}$ Engl J Med. 2010;362(8):697-706.

5. National Institute of Allergy and Infectious Diseases. Questions and Answers: The CAMELIA clinical trial. Available at www.niaid.nih.gov/news/QA/Pages/CAMEL IAqa.aspx. Accessed on January 31, 2011.

6. Panel on Antiretroviral Guidelines for Adults and Adolescents. Department of Health and Human Services. Available at http://www.aidsinfo.nih.gov/ContentFiles/ AdultandAdolescentGL.pdf. Accessed on August 31, 2011.

7. Manosuthi W, Sungkanuparph S, Tantanathip P, Lueangniyomkul A, Mankatitham W, Prasithsirskul W, et al. A randomized trial comparing plasma drug concentrations and efficacies between 2 nonnucleoside reverse-transcriptase inhibitor-based regimens in HIVinfected patients receiving rifampicin: the N2R Study. Clin Infect Dis. 2009;48:1752-9.

8. Shah I, Swaminathan S, Ramachandran G, Kumar AK, Goray A, Chaddha U, et al. Serum nevirapine and efavirenz concentrations and effect of concomitant use of rifampicin in HIV infected children on antiretroviral therapy. Indian Pediatr. 2011;48:943-7.

9. Ren Y, Nuttall JJ, Eley BS, Meyers TM, Smith PJ, Maartens $\mathrm{G}$, et al. Effect of rifampicin on efavirenz pharmacokinetics in HIV-infected children with tuberculosis. J Acquir Immune Defic Syndr. 2009;50: 439-43.

10. Rotger M, Colombo S, Furrer H, Bleiber G, Buclin T, Lee $\mathrm{BL}$, et al. Influence of CYP2B6 polymorphism on plasma and intracellular concentrations and toxicity of efavirenz and nevirapine in HIV-infected patients. Pharmacogenet Genomics. 2005;15:1-5. 